\title{
Application of CEEMD-SVM in Rolling Bearing Fault Recognition
}

$$
\text { Xiang } \mathrm{Li}^{1, \mathrm{a}} \text { and Feng Ding }{ }^{2, \mathrm{~b}^{*}}
$$

${ }^{1,2}$ Department of Mechanical and Electronic Engineering, Xi'an Technological University, Xi'an, China

axatuxiangli@163.com, bdfeng@xatu.edu.cn

*The corresponding author

Keywords: Complementary ensemble empirical mode decomposition; Support vector machine; Rolling bearing; Fault recognition

\begin{abstract}
To extract effective fault features from the nonstationary vibration signals of rolling bearing, this paper provides a fault recognition method by using complementary ensemble empirical mode decomposition (CEEMD) and support vector machine (SVM). Firstly, CEEMD is applied to process vibration signals of rolling bearing. Then some time domain features of the first several intrinsic mode functions (IMFs) are calculated to construct feature set, and it is utilized as the input of SVM. Finally, different fault states of rolling bearing are classified through SVM. The experiment results indicate that the presented method is effective, and it can significantly improve the classification accuracy of fault recognition.
\end{abstract}

\section{Introduction}

Rolling bearing is one of the most important parts of rotor system, and a variety of fault states are produced in the process of working. The fault severity is worse with the long running time of rolling bearing. Due to the complex structure of rotor system, many vibration sources are generated. The signals which are produced by these vibration sources are collected through different transmission paths. Therefore the acquired vibration signals of rolling bearing are no stationary [1].

Empirical mode decomposition (EMD) is proposed, and it can decompose signals into a series of intrinsic mode functions (IMFs) [2, 3]. However, the problems including mode aliasing and over decomposition are produced. In order to overcome these disadvantages, Yeh et al [4] put forward the complementary ensemble empirical mode decomposition (CEEMD) for processing no stationary signals. In this method, two sets of white noise signals with positive and negative signs are added into original signals. CEEMD is able to remove the residual noise in the IMFs and computational efficiency can also be enhanced $[5,6]$.

In this paper, CEEMD is applied to process vibration signals of rolling bearing with different fault states. Time domain features of the first several IMFs are calculated to construct feature set. Eventually support vector machine (SVM) is used to classify rolling bearing fault states.

\section{Extraction of Time Domain Features Based on CEEMD}

CEEMD Algorithm. CEEMD is a noise assisted method to solve the problem of mode aliasing by adding positive and negative white noises to signals. It can remove the residual noise in the reconstructed signals and lower computational burden. The decomposition process is consisted of the following three steps.

(1)Adding white noise signals with opposite signs to original signal $x(t)$. The obtained two signals are as:

$\left\{\begin{array}{l}x_{1}^{+}(t)=x(t)+w_{1}(t) \\ x_{1}^{-}(t)=x(t)-w_{1}(t)\end{array}\right.$

(2)The two signals are decomposed by EMD separately, and two sets of components 
$\mathrm{IMF}_{+1 i}, \mathrm{IMF}_{-1 i}$ are achieved. They represent the $i$ th components which are generated by decomposing the signals with the first positive and negative white noise.

(3)Repeating above steps with $n$ times, and $n$ sets of components are obtained.

$$
\left\{\begin{array}{l}
\mathrm{IMF}_{+j i}, j=1,2, \cdots n \\
\mathrm{IMF}_{-j i}, j=1,2, \cdots n
\end{array}\right.
$$

(4)The $n$ sets of components are processed by averaging operation, and the final decomposition results are achieved.

$$
\mathrm{IMF}_{i}=\frac{1}{2 n} \sum_{j=1}^{n}\left(\mathrm{IMF}_{+j i}+\mathrm{IMF}_{-j i}\right)
$$

Extraction of Time Domain Features. When different rolling bearing fault states emerge, the amplitude and waveform of time domain signals are also different [7]. Thus 10 kinds of time domain features of the first several IMFs are calculated to construct feature set. These features are calculated as the description of Table $1, x_{i}$ is the time domain waveform sequence of rolling bearing vibration signals.

\begin{tabular}{|c|c|c|c|}
\hline Features & Mathematical expression & Features & Mathematical expression \\
\hline $\begin{array}{l}\text { Margin } \\
\text { index }\end{array}$ & $x_{m a}=\frac{\max \left(\left|x_{i}\right|\right)}{\left(\frac{1}{N} \sum_{i=1}^{N} \sqrt{\left|x_{i}\right|}\right)^{2}}$ & Peak value & $x_{p}=\max \left(\left|x_{i}\right|\right)$ \\
\hline $\begin{array}{c}\text { Root } \\
\text { amplitude }\end{array}$ & $x_{r a}=\frac{\sum_{i=1}^{N} \sqrt{\left|x_{i}\right|}}{N}$ & $\begin{array}{l}\text { Root mean } \\
\text { square value }\end{array}$ & $x_{r m s}=\sqrt{\frac{\sum_{i=1}^{N} x_{i}^{2}}{N}}$ \\
\hline $\begin{array}{l}\text { Standard } \\
\text { deviation }\end{array}$ & $\sigma=\sqrt{\frac{\sum_{i=1}^{N}\left(x_{i}-\bar{x}\right)^{2}}{N-1}}$ & Skewness & $x_{s k e}=\frac{1}{N-1} \sum_{i=1}^{N}\left(\frac{x_{i}-\bar{x}}{\sigma}\right)^{3}$ \\
\hline Kurtosis & $x_{k}=\frac{1}{N-1} \sum_{i=1}^{N}\left(\frac{x_{i}-\bar{x}}{\sigma}\right)^{4}$ & $\begin{array}{l}\text { Waveform } \\
\text { index }\end{array}$ & $x_{\text {sha }}=\frac{\sqrt{\frac{1}{N} \sum_{i=1}^{N} x_{i}^{2}}}{\frac{1}{N} \sum_{i=1}^{N} x_{i}}$ \\
\hline $\begin{array}{l}\text { Peak } \\
\text { index }\end{array}$ & $x_{c}=\frac{\max \left(\left|x_{i}\right|\right)}{\sqrt{\frac{1}{N} \sum_{i=1}^{N} x_{i}^{2}}}$ & Pulse index & $x_{i}=\frac{\max \left(\left|x_{i}\right|\right)}{\frac{1}{N} \sum_{i=1}^{N} x_{i}}$ \\
\hline
\end{tabular}

Table 1 Time domain features

\section{Rolling Bearing Fault Recognition}

The feature set of training samples is fed into SVM [8-10], and cross validation method is utilized to optimize the parameters of the classifier. Then the obtained SVM is applied to accurately classify different fault states of rolling bearing.

\section{Experiment Validation}

Feature Extraction of Rolling Bearing Based on CEEMD. Experimental data used in this paper 
is from the Case Western Reserve University. Rolling bearing vibration signals with 6 kinds of fault states are separately processed by CEEMD. The sampling frequency of vibration signals is $12 \mathrm{KHz}$, and there are 4096 data points in each sample. The specific description of samples is presented in Table 2. The first 8 IMFs are selected to calculate the time domain features. Fig. 1 is the first 8 IMFs of rolling bearing inner race fault signals and its fault severity is $0.007 \mathrm{in}$.

In the meantime, 10 kinds of time domain features of the first 8 IMFs are calculated to construct feature set which containing 80 feature indicators. Then the feature set is fed into SVM for classifying rolling bearing fault states.

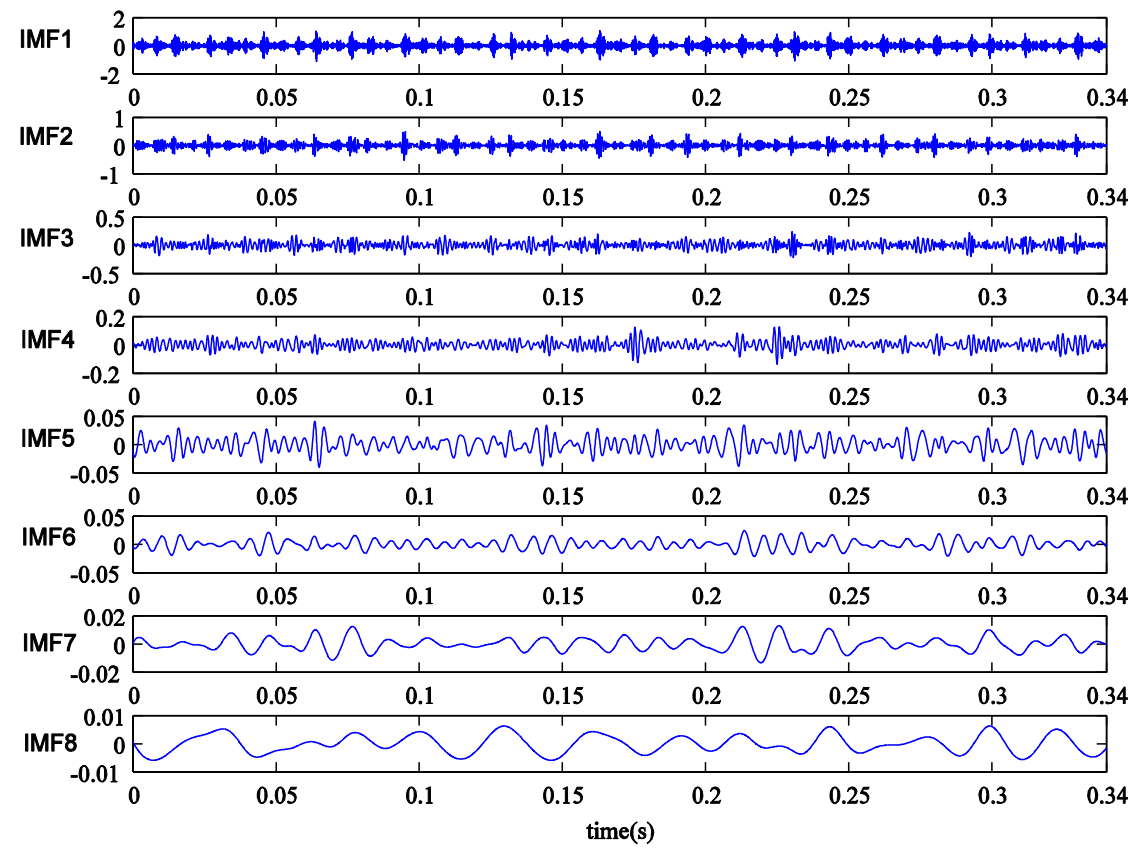

Figure 1. The first 8 IMFs of rolling bearing inner race fault signals

Table 2 Specific description of rolling bearing samples

\begin{tabular}{cccc}
\hline Fault states [in] & $\begin{array}{l}\text { Number of training } \\
\text { samples }\end{array}$ & $\begin{array}{l}\text { Number of testing } \\
\text { samples }\end{array}$ & Labels \\
\hline Inner race 0.007 & 15 & 15 & 1 \\
Inner race 0.021 & 15 & 15 & 2 \\
Outer race 0.007 & 15 & 15 & 3 \\
Outer race 0.021 & 15 & 15 & 4 \\
Rolling element 0.007 & 15 & 15 & 5 \\
Rolling element 0.021 & 15 & 15 & 6 \\
\hline
\end{tabular}

Classification of Rolling Bearing Fault States. The time domain feature set of training samples is applied to train the SVM classifier. Cross validation method is used to optimize parameters of SVM. Specifically, the values of kernel function parameter $g$ and penalty factor $c$ are set in the range of $2^{-5} \square 2^{5}$. The highest classification accuracy is $92.2222 \%$ while $g=0.0078, c=512$. Then the trained SVM is utilized to classify the testing samples of rolling bearing. Fig. 2 is the classification results, and it can be seen that the classification accuracy reaches $94.4444 \%$. 


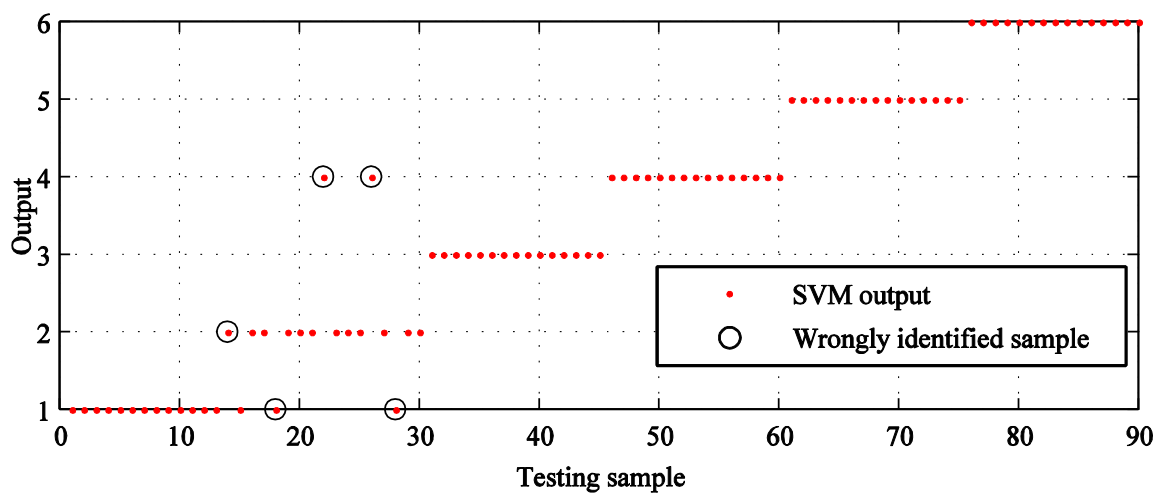

Figure 2. Fault recognition results of rolling bearing based on CEEMD

Analysis of Results. Traditional EMD is also applied to process vibration signals of rolling bearing, and the same time domain feature set is achieved. The classification results of SVM are described in Figure 3.

It can be seen that the classification effect is not ideal. The accuracy of fault recognition decreases to $87.7778 \%$. Therefore, we can find that CEEMD is more effective and suitable for rolling bearing fault recognition. The classification accuracy can be greatly enhanced.

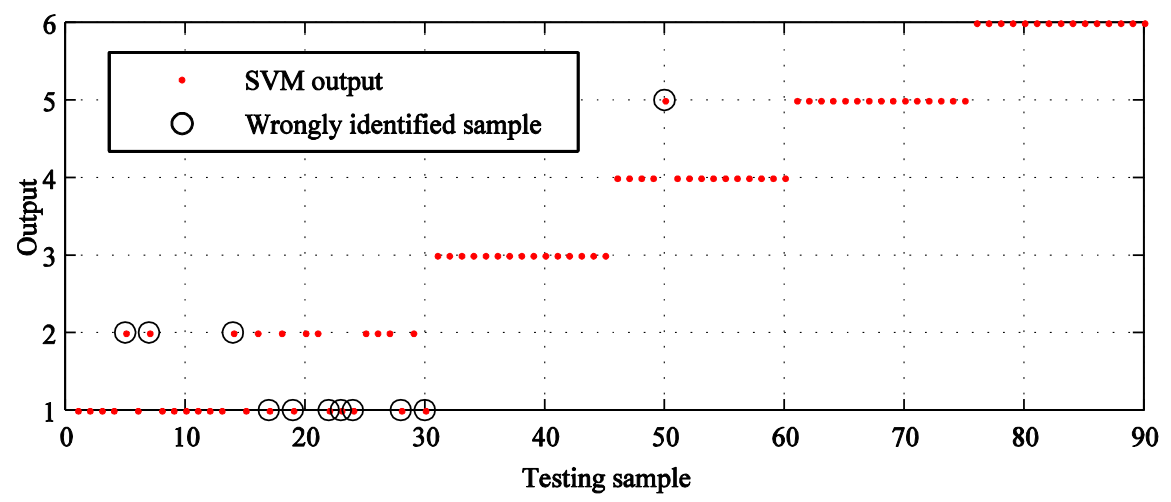

Figure 3. Fault recognition results of rolling bearing based on traditional EMD

\section{Summary}

In order to accurately extract fault feature information, this paper proposes CEEMD-SVM method to classify different fault states of rolling bearing. CEEMD is able to solve the problems including mode aliasing and over decomposition which exist in the traditional EMD. In the meantime, 10 kinds of time domain features which are calculated from the first several IMFs can effectively reveal fault states of rolling bearing. Compared with traditional EMD, the experiment results demonstrate that the presented method has higher classification accuracy and better robustness.

\section{Acknowledgements}

This research is financially supported by the National Science Foundation of China (grant no. 51275374) and the Fund Project of Science \& Technology on Reliability \& Environmental Engineering Key Laboratory.

\section{References}

[1] H. C. Wang, J. Chen and G. M. Dong: Feature extraction of rolling bearing's early weak fault based on EEMD and tunable Q-factor wavelet transform, Mechanical Systems and Signal Processing, Vol.48 (2014) No.1-2, p.103-119. 
[2] Y. Yang, D. J. Yu and Chen J. S: Rolling bearing fault diagnosis method based on EMD and neural network, Journal of Vibration and Shock, Vol.24 (2005) No.1, p.85-88, (In Chinese).

[3] D. J. Yu: The Hilbert-Huang transform method of mechanical fault diagnosis (Science Publications, China 2007), p.56-62.

[4] J. R. Yeh, J. S. Shieh and N. E. Huang: Complementary ensemble empirical mode decomposition: A novel noise enhanced data analysis method, Advances in Adaptive Data Analysis, Vol.2 (2010) No.2, p.135-156.

[5] T. T. Zhou, X. M. Zhu and W. C. Peng, et al: A wavelet threshold denosing method for fault data based on CEEMD and permutation entropy, Journal of Vibration and Shock, Vol.34 (2015) No.23, p.207-210, (In Chinese).

[6] L. Y. Zhao, W. Yu and R. Q. Yan: Gearbox fault diagnosis using complementary empirical mode decomposition and permutation entropy, Shock and Vibration, Vol.2016 (2016), p.1-8.

[7] J. X. Qu, F. Y. Yang and Z. S. Zhang, et al: Identification method for structural damage based on acoustic signal, Journal of Vibration, Measurement and Diagnosis, Vol.34 (2014) No.4, p.638-643, (In Chinese).

[8] Y. B. Li, M. Q. Xu and Y. Wei, et al: A new rolling bearing fault diagnosis method based on multiscale permutation entropy and improved support vector machine based binary tree, Measurement, Vol.77 (2016), p.80-94.

[9] Q. Hu, Z. J. He and Z. S. Zhang, et al: Fault diagnosis of rotating machinery based on improved wavelet package transform and SVMs ensemble, Mechanical Systems and Signal Processing, Vol.21 (2007) No.2, p.688-705.

[10] A. Widodo, B. S. Yang: Support vector machine in machine condition monitoring and fault diagnosis, Mechanical Systems and Signal Processing, Vol.21 (2007) No.6, p.2560-2574. 